\title{
Treatment Enhances Ultradian Rhythms of CSF Monoamine Metabolites in Patients with Major Depressive Episodes
}

\author{
Ronald M Salomon*,', John S Kennedy², Benjamin W Johnson ${ }^{3}$, Jennifer Urbano Blackford', \\ Dennis E Schmidt', Joseph Kwentus ${ }^{4}$, Harry E Gwirtsman', John F Gouda ${ }^{5}$ and Richard G Shiavi ${ }^{5}$ \\ 'Department of Psychiatry, Vanderbilt University School of Medicine, Nashville, TN, USA; 'Department of Psychiatry, Indiana University School of \\ Medicine, Indianapolis, IN, USA; ${ }^{3}$ Department of Anesthesiology, Vanderbilt University School of Medicine, Nashville, TN, USA; ${ }^{4}$ Department of \\ Psychiatry, University of Mississippi School of Medicine, Jackson, MS, USA; ${ }^{5}$ Department of Biomedical Engineering, Vanderbilt University, \\ Nashville, TN, USA
}

\begin{abstract}
Unipolar and bipolar depressions show abnormal behavioral manifestations of ultradian (less than $24 \mathrm{~h}$ ) rhythms, but abnormal rhythms of the central neurotransmitters thought to be important for depression pathophysiology (eg dopamine (DA) and serotonin (5-HT)) have not been shown in this time frame. Since antidepressant treatments normalize disrupted rhythms in depression (eg rapid-eyemovement sleep and hormonal rhythms), we hypothesized that depression-related changes in ultradian oscillations of DA and 5-HT might be revealed during antidepressant treatment. Cerebrospinal fluid (CSF) samples collected ql0 min for $24 \mathrm{~h}$ in 13 patients experiencing major depressive episodes (MDE) before and after treatment for 5 weeks with sertraline or bupropion were assayed for levels of homovanillic acid (HVA) and 5-hydroxyindoleacetic acid (5-HIAA), and their ratio was calculated. Data were analyzed in the frequency domain using Fourier transforms and multivariate permutation testing. Antidepressant treatments were associated with decreased variance for 5-HIAA, increased variance for HVA, and markedly increased variance for the HVA: 5-HIAA ratio ( $p<0.05$, $p<0.02$, and $p<0.003$, respectively). With treatment, the correlations between 5-HIAA and HVA weakened $(p=0.06)$. Power spectral density (PSD - the Fourier magnitude squared) of the 5-HIAA signals at periods of 1.75 and $3.7 \mathrm{~h}$ (both $p<0.05)$ decreased, while circadian cycling of HVA levels $(p<0.05)$ and of the ratio $(p<0.005)$ increased after treatment. The PSD of the full-length HVA: 5-HIAA ratio series after treatment increased in rapid variability $(20-103$ min periods, $p<0.05)$. Spectrographic windowing demonstrated a focal span of enhanced HVA:5-HIAA ratio variability following antidepressant treatment, in an approximately 84-min period through the evening $(p<0.05)$. Periodic neurotransmitter relationships in depressed patients were altered by treatment in this analysis of a small data set. This may represent a baseline abnormality in the regulation of periodic functions involved in the depression pathophysiology, but it could also be due to an unrelated antidepressant effect. Further studies including comparisons with healthy subject data are in progress. Neuropsychopharmacology (2005) 30, 2082-209I. doi:I 0.1038/sj.npp. I 300746; published online 27 April 2005
\end{abstract}

Keywords: depression; biological rhythms; 5-hydroxyindoleacetic acid (5-HIAA); homovanillic acid (HVA); time series; multivariate permutation test

\section{INTRODUCTION}

Diminished variability in thought, behavior, and mood are distinguishing features of major depression that are reliably detected in serial measures or ratings over periods of time shorter than $24 \mathrm{~h}$. Healthy individuals experience variable moods over time and attribute them to elective thoughts or events in the environment, while in depression the mood changes are felt to be 'uninfluenceable' (Wefelmeyer and

\footnotetext{
*Correspondence: Dr RM Salomon, Department of Psychiatry, Vanderbilt Univ. School of Medicine, 1500 2IST Avenue South, Suite VAV-2200 Psychiatry, Nashville, USA, Tel: +6I5 322 0387, Fax: + 615343 7868, E-mail: ron.salomon@vanderbilt.edu

Received 13 October 2004; revised 15 February 2005; accepted 15 March 2005

Online publication: 22 March 2005 at http://www.acnp.org/citations/ Npp032205040475/default.pdf
}

Kuhs, 1996). Studies using high-frequency sampling to investigate neurobiological variability in depression are needed. Cross-sectional data across a population at a single time point may not reliably capture mood since depressed people often smile for a moment just as happy people sometimes frown. Time series information from EEG lacks selective neurotransmitter information and is limited to cortical activation. Most studies of neurochemical changes in depression have utilized only single time values as the primary outcome measures, for example, levels of cerebrospinal fluid (CSF) metabolites, neuroendocrine outputs as area-under-the-curve (AUC), PET uptake or ligand binding, or average of activities in fMRI. Geracioti et al $(1992,1997,1998)$ have previously shown the safety and utility of high-frequency CSF sampling to study dynamic neurochemical changes in depression and other disorders. 
Altered circadian periodicities in mood behaviors (Kraepelin, 1913) were recognized before central neurochemical measurements were possible. Disrupted biological rhythms have been hypothesized to underlie affective dysregulation (Healy, 1987; Ehlers et al, 1988). Phase-advanced temperature profiles and onsets of rapid-eye-movement (REM) sleep relative to sleep schedules in depression prompted treatment trials using a shift of the sleep schedule forward by $6 \mathrm{~h}$ to correct the shift in temperature and REM rhythms (Wehr et al, 1979). Alternatively, biological rhythm disruptions and depression after psychosocial losses have also been linked to changes in social zeitgebers resulting from such losses (Ehlers et al, 1988).

Neurophysiologic ultradian rhythms are diminished in depression. Ultradian oscillatory patterns in electrical (EEG) activity (Polich, 1997) change with mood (Cazard et al, 1992; Hayashi et al, 1994). EEG asymmetry across hemispheres (Klein and Armitage, 1979; Shannahoff-Khalsa, 1993) and intra-and interhemispheric coherence losses (Armitage et al, 1999) demonstrate dysfunctional oscillatory activity in the cortex in depression (Armitage et al, 2000). EEG dynamical complexity (nonlinear forecasting and entropy) shows lower entropy in depression and an association between poor recovery of these measures during treatment and progression and recurrences of the illness (Pezard et al, 1996).

Circadian mood rhythms (Carpenter et al, 1986; WirzJustice, 2000) and the predictions of treatment outcome based on pretreatment diurnal patterns (Carpenter et al, 1986; Haug and Wirz-Justice, 1993) suggest that rhythmic variability is important for healthy emotional function. Evidence suggests potential mechanisms and effects of rhythmic variability, for example, tryptophan hydroxylase function shows dynamic variability in serotonin (5-HT) synthesis (Knapp and Mandell, 1983), and in depression hypothalamic peptides modulate EEG complexity during sleep (Ehlers and Kupfer, 1987). Peripheral 5-HT function, as reflected in platelet activity, shows circadian disturbances in depression (Oxenkrug, 1979) and schizophrenia (Rao et al, 1994). Circadian neurochemical changes have been related to pacemaker effects of the suprachiasmatic nucleus (Bunney and Bunney, 2000).

However, the existence of a true basic rest-activity cycle (Kleitman, 1982) or an ultradian neurochemical oscillator remains controversial because ultradian rhythms in neurochemical activity remains under-reported and poorly understood. Recently, measures from samples taken every $10 \mathrm{~min}$ for $24 \mathrm{~h}$ from 12 healthy subjects showed variability of both the serotonin precursor (tryptophan, TRP) and its major metabolite (5-HIAA) in lumbar CSF that significantly exceeded variability of the assay used (both $p<0.0001 ; 25.4$ vs $7.9 \%$ and 32 vs $7.0 \%$, respectively). A strong circadian variability accounted for much of the TRP variance but was not a significant component of the 5-HIAA variance (Kennedy et al, 2002). Ultradian rhythms accounted for much of the remaining variance, with prominent $12 \mathrm{~h}, 3 \mathrm{~h}$, $90 \mathrm{~min}$, and $53 \mathrm{~min}$ wavelengths in the 5-HIAA time series (accounting for $69 \%$ of the variance), and with 12 and $3 \mathrm{~h}$ wavelengths in HVA levels (accounting for $73 \%$ of the variance) in healthy subjects (H Gwirtsman, personal communication). Thus, intensive and prolonged sampling of healthy human lumbar CSF has shown neurochemical ultradian rhythms, but changes in these rhythms have not previously been shown in depression.

Serial sampling studies have examined levels of the individual neurotransmitter metabolites. The HVA : 5-HIAA ratio has been used previously in single sample studies to indicate relative transmitter turnover rates. Antidepressant treatments increased this ratio (DeBellis et al, 1993), largely the result of diminished 5-HIAA levels in the denominator, but differently among various treatments (Risby et al, 1987) and, in pretreatment measures, predicting clinical outcome (Carpenter et al, 2004). This ratio has been shown to be remarkably constant and unaffected by the stress of the lumbar puncture (Geracioti et al, 1998; Hill et al, 1999). In a time series, changes in the ratio may reflect the independence of the dopamine (DA) and 5-HT systems. Low local variability in the ratio (eg, a flat profile) would suggest strong positive interactions or an external mechanism that coregulates or synchronizes the systems. Increased variability in the ratio would be anticipated with greater independence or with negative interactions. Time-dependent ultradian changes in the ratio differ from simple correlations, which only give an average 'independence' over the entire signal.

Preclinical evidence of mechanisms that could cause diminished neurobiologic rhythms in depression is abundant. Covariability of monoaminergic function varies throughout the $24 \mathrm{~h}$ cycle. Strong intercorrelations of dorsal raphe 5-HT and locus coeruleus norepinephrine concentrations during the day were diminished at night (Agren et al, 1986). Expression of the 5-HT transporter fluctuates over time and affects citalopram binding, sleep behaviors, brain 5-HT levels, 5- $\mathrm{HT}_{1 \mathrm{~A}}$ receptors, and 5-HIAA levels (Fabre et al 2000). Both citalopram binding at the serotonin transporter (Kulikov et al, 1997) and 5- $\mathrm{HT}_{2 \mathrm{C}}$ receptor RNA expression (Holmes et al, 1997) increase significantly during morning hours. Monoamine function varies diurnally in rodent hypothalamus slices (Blier et al, 1989) and 5HIAA rhythms vary regionally in rodent microdialysis studies (Nakayama, 2002).

Specific receptor subtypes control circadian events. The $5-\mathrm{HT}_{1 \mathrm{~A}}$ partial agonist, gepirone, induces phase-advances in circadian activity rhythms (Van Reeth et al, 1999). The 5$\mathrm{HT}_{7}$ receptor also may mediate phase-advances in the suprachiasmatic nucleus in the rat and is downregulated in response to fluoxetine. Activators of $5-\mathrm{HT}_{7}$ include a variety of antidepressants (Lovenberg et al, 1993; Mullins et al, 1999). Periodic cell function determinants, possibly including cyclin-dependant kinases, may point to novel treatments for psychiatric disorders (Chergui et al, 2004). 'Healthy' oscillatory function would appear to be important since neurotrophic activations are enhanced by oscillations in transmitter levels (Nishi et al, 2000).

Clinical studies have identified altered circadian neurochemical rhythms in depression, with diminished variability of plasma amino-acid monoamine precursors (Birkmayer and Linauer, 1970) and plasma monoamine metabolites (Riederer et al, 1974). Periodicity of hypocretin levels is diminished in depression (Prospero-Garcia et al, 1999; Salomon et al, 2003). MAOI effects on rest-activity cycles are controlled in part by light schedules (Duncan et al, 1998). In view of these findings, lost efficacy with chronic antidepressant use (Cassano and Fava, 2004) could be due 
to recurrent maladaptive network attractors (boundarylimited looping patterns of activity), creating an overly constrained ultradian cycle.

Antidepressant treatments restore normal cycles of mood (Jouvent et al, 1998), REM sleep (Thase et al, 1998), EEG $\theta$ frequencies (Knott et al, 1996), rest-activity cycles (Raoux et al, 1994; Duncan et al, 1998), and hormonal rhythms (see review, Duncan, 1986). To test a hypothesis that ultradian neurochemical rhythms would be affected by antidepressants, CSF samples at 10 -min intervals for $24 \mathrm{~h}$ were collected from depressed patients and examined for changes in spectral (oscillatory frequency) features before and after antidepressant treatment.

Frequency domain analyses of neurochemical time series data should help to refine and reconcile models of neurochemical dysfunction in depression, for example, the catecholamine deficit (Schildkraut, 1965) or the phaseadvance (Wehr et al, 1980) models. The Fourier transformation displays the relative strengths of various frequencies in a set of coefficients for specific wavelengths, analogous to marking signal strengths along an FM radio dial. Validity of the transformed data is established by its mathematical uniqueness, the independence (orthogonality) of each frequency coefficient, and the faithful reproduction of the original signal by the inverse transform with minimal gains or losses of information. The power spectral density (PSD), which is the square of the Fourier transform magnitude, is more often implemented. Spectrograms are serial PSDs from shorter segments of the sample that are lined up consecutively by time period along the time axis. Paired Fourier or PSD coefficients in the spectrogram can be compared statistically with multivariate permutation testing. We used these methods to test the hypothesis that power in a specific bandwidth or limited number of bandwidths would change after treatment in patients with major depressive episodes in a within-subjects design. The data suggest that changes in periodic patterns in neurochemical output may be important markers of neural network dysfunction in major depression.

\section{MATERIALS AND METHODS}

\section{Patients}

All studies were approved by the Vanderbilt IRB. After extensive individual discussions to obtain written informed consent, subjects were admitted to the Vanderbilt General Clinical Research Center (GCRC). A total of 15 depressed patients (10 female, five male, ages 25-60 years, mean 37.7 years) met all criteria below and DSM-IV (APA, 1994) criteria for Major Depressive Episode (Major Depressive Disorder or Bipolar Disorder, Depressed). Diagnoses of major depression were established by SCID-P (First et al, 1996a). Severity of depression was quantified by mood ratings (Hamilton Rating Scale for Depression, HRSD, from the Yale Depression Inventory, Mazure et al, 1986). Both were confirmed in a clinical interview by a boarded psychiatrist (RMS). Exclusions for severe personality disorders were assured by SCID-II (First et al, 1996b). Medical history review, physical exam, ECG, and laboratory evaluations showed that all subjects were free of other significant abnormalities. Patients were free of psychotropic medications and substance abuse for 2 and 6 months, respectively, and were free of all other medications for at least 1 month, including OTC analgesics. Potentially suicidal patients were excluded to avoid delaying treatment, because of invasive study risks, and because of potentially inappropriate motivation for participation. Adequate volitional and cognitive capacity was required. Data from 13 patients, none of whom had a lifetime history of major substance abuse, were analyzed (Data from one male was excluded because of a catheter occlusion. One female was excluded because she was maintained on lithium continuously and her data were extreme outliers, to be reported elsewhere.).

\section{Procedure}

Diet for $72 \mathrm{~h}$ before and $24 \mathrm{~h}$ during collections was caffeinefree and monoamine-balanced. Patients were supine overnight before catheter placement. Lights out was from 2230 to 0600 hours, interrupted briefly by flashlight-only entries for sample retrieval every $30 \mathrm{~min}$ and tympanic temperature at 0200 hours. Tympanic temperature was also determined every $2 \mathrm{~h}$ while awake. Daytime napping was discouraged.

CSF for monoamine metabolite assays was obtained by lumbar catheterization for $24 \mathrm{~h}$. A $20 \mathrm{~g}$ catheter from the L45 interspace was connected to a peristaltic pump for continuous collection of $1-\mathrm{ml}$ CSF samples every $10 \mathrm{~min}$ in a refrigerated fraction collector $\left(\leqslant 5^{\circ} \mathrm{C}\right)$. Samples were frozen on dry ice within $30 \mathrm{~min}$ of collection and later stored at $-70^{\circ} \mathrm{C}$. High pressure liquid chromatography (HPLC) with electrochemical detection (Schmidt et al, 1990) was used to determine concentrations of tryptophan (the amino-acid precursor of 5-HT, TRP), homovanillic acid (the DA metabolite, HVA), 5-hydroxyindoleacetic acid (the 5-HT metabolite, 5-HIAA), and the metabolite ratio (HVA:5HIAA). Studies measuring the HVA:5-HIAA ratio have been reported previously (Engstrom et al, 1999; Risby et al, 1987) and were also determined in these sets.

The CSF collection procedure was repeated after treatment with antidepressants, continuing antidepressant administration throughout the second CSF collection at week 5 and then through week 8 to the treatment end point. Except moderate to severe headache after half of the catheterizations, procedures were well tolerated. Blood patches were performed for nine of the 30 studies and all headaches resolved. There were no serious adverse events or long-term sequellae.

\section{Mood Outcome Measures}

Mood response to treatment was defined a priori as a $50 \%$ decline in 17-item HRSD. HRSD scores at screening, twice daily during each admission, and weekly at out patient visits were summarized, with response status determined at week 8 .

\section{Antidepressant Treatment}

Two medications with divergent transporter selectivities were used to test whether each would equally affect neurochemical variance. If the primary hypothesis that variance in neurochemical function is an attribute of health 
Table I Mean Area-Under-the-Curve (AUC) of Power Spectral Densities (PSD) for Full Spectra from Full 24-h Data Set

\begin{tabular}{|c|c|c|c|c|c|c|}
\hline & Pretreatment mean & Pretreatment SD & Post-treatment mean & Post-treatment SD & $t$ & $p$ \\
\hline 5-HIAA* & 7.74 e7 & $6.92 \mathrm{e} 7$ & 4.29 e7 & 2.39 e7 & -2.217 & 0.047 \\
\hline TRP & I.01 elo & 8.48 e 9 & 1.32 elo & 1.16 e 10 & 0.804 & 0.437 \\
\hline HVA* & 3.63 e8 & 2.09 e8 & 6.57 e8 & 5.04 e8 & 2.701 & 0.019 \\
\hline HVA/5-HIAA*** & 2.02 e 4 & $1.36 \mathrm{e} 4$ & $6.92 \mathrm{e} 4$ & 4.28 e 4 & 3.891 & 0.002 \\
\hline
\end{tabular}

$* p<0.05 ; * * 0<0.005$, paired $t$-tests, two-tailed.

Spectral power from $24 \mathrm{~h}$ of CSF samples with 10 min intersample interval, $n=13$. Decline in 5-HIAA spectral power density reflects decreased variance after treatment, and increased HVA and ratio spectral power density (HVA:5-HIAA) reflect increased variance with treatment.

Values in bold are for $p<0.05$.

Table 2 Change in Frequency Response with Treatment — t-Values for Area-Under-the Curve (AUC) in Five Bandwidths from 24-h Serial Sampling Data (Samples Every 10 min, I 44 Samples per Collection)

\begin{tabular}{lccccccc}
\hline Band & PSD coeffs. & Period length (time per cycle) & Label & 5-HIAA & TRP & HVA & Ratio \\
\hline 1 & $1-7$ & Infinite $\rightarrow 4 \mathrm{~h}$ & $24 \mathrm{~h}$ & -1.384 & 0.575 & $2.447 *$ \\
2 & $7-8$ & $4 \rightarrow 3.4 \mathrm{~h}$ & $3.7 \mathrm{~h}$ & $-2.547 *$ & 0.954 & $-0.24 \mid$ & $3.560^{* * *}$ \\
3 & $8-14$ & $3.4 \rightarrow 1.85 \mathrm{~h}$ & $2.5 \mathrm{~h}$ & -1.460 & 0.803 & 0.250 & 1.098 \\
4 & $14-15$ & $1.85 \rightarrow \mid .7 \mathrm{~h}$ & $1.75 \mathrm{~h}$ & $-2.406 *$ & 1.58 & 0.767 & 1.958 \\
5 & $16-72$ & $1.7 \rightarrow 0.3 \mathrm{~h}$ & $0.75 \mathrm{~h}$ & -1.246 & 1.282 & 1.884 & $2.843 *$ \\
\hline
\end{tabular}

$* p<0.05 ; * * p<0.005$.

Calculated $t$-values for area-under-the curve (AUC) of five bandwidths of the power spectral density (PSD) from 24-h serial sampling for monoamine metabolite levels (samples every $10 \mathrm{~min}, 144$ samples per collection, no windowing). Change with treatment shows significantly enhanced power in the slowest frequencies (24-h to 4-h periods) for HVA and the HVA:5-HIAA ratio, but in moderate frequencies (4- to 3.5-h periods and III-to I03 min periods) for the 5-HIAA fluctuations alone.

holds true, then any effective antidepressant treatment would be expected to enhance neurochemical variance. A single medication (sertraline) was given to the first five patients, leaving open the question of whether a singular $v s$ multiple drug mechanisms would be critical to enhancements of variance. The randomization procedure (with enrollment sequence double blind to future assignment) was added to the protocol at that point, adding four more patients on sertraline and four on bupropion to the total set. Open-label sertraline treatment $(50 \mathrm{mg} /$ day week one, then $100 \mathrm{mg}$ daily) or bupropion treatment (150 mg slow-release/ day week 1 , then $150 \mathrm{mg}$ BID) began 3 days after the first CSF collection.

\section{Analysis}

Time series data were examined initially for treatmentassociated changes in means, standard deviation, and overall variance. Frequency domain exploration used the Fourier transform of the full-length data set (Table 1). The Fourier coefficients were then averaged across individuals and post- minus pretreatment differences were calculated. Frequency coefficients of the PSD from the 24-h time series were derived without log expansion, and five frequency bandwidths (Table 2) were assessed using an AUC in order to compare groups of adjacent coefficients, accommodating slight individual differences in peak frequency components.

Spectrogram analyses allow testing for isolated periodicity within shorter time spans than the full 24-h data set. The data were windowed in time in order to uncover specific times of day when these changes in periodicity characteristics might be most prominent. Spectrograms used data windows of 52 points $=8.67 \mathrm{~h}$, a 51 -point overlap, with PSDs calculated after local linear-detrending, hamming-windowing of each window segment, and zeropadding to 64 points $(10.7 \mathrm{~h})$. A wide range of window lengths was tested to assure that similar final results would be obtained and that the particular window choice was not spurious. Trapezoidal areas-under-the-curve (AUC) created 10 evenly spaced PSD bandwidths for spectrogram analysis (Table 3).

Missing data were less than $3 \%$ of the total and were manually interpolated prior to analysis. Cohen's effect size (d) was determined and is reported for the main findings (Cohen, 1992). Pretreatment and post-treatment data were compared using multivariate permutation testing. Multivariate permutation paired $t$-tests (MPT, Blair and Karniski, 1994; Blackford, 2002) were performed on the difference spectrogram in both frequency and time simultaneously while controlling for family-wise error. All analyses were performed using the open source-code package, $R$ ( $\mathrm{R}$ ver. 1.8.1, 2003). All statistics are two-tailed with $\alpha=0.05$.

\section{RESULTS}

\section{Overview}

Following antidepressant treatment, both the variance and overall spectral power of the HVA : 5-HIAA ratio increased. Spectrograms also changed after treatment, showing increased periodicity that was maximal at a cycle length of about $84 \mathrm{~min}$ and lasted about $2.8 \mathrm{~h}$ in the evening. This 
Table 3 Bandwidths and Corresponding Coefficients for Spectrograms

\begin{tabular}{lll}
\hline Bandwidth & Coefficient number & Period length \\
\hline 1 & $1: 2$ & Infinity- $10.7 \mathrm{~h}$ \\
2 & $2: 3$ & $10.7-5.3 \mathrm{~h}$ \\
3 & $3: 5$ & $5.3-2.7 \mathrm{~h}$ \\
4 & $5: 7$ & $2.7-1.7 \mathrm{~h}$ \\
5 & $7: 10$ & $1.7-1.2 \mathrm{~h}$ \\
6 & $10: 13$ & $1.2 \mathrm{~h}-53 \mathrm{~min}$ \\
7 & $13: 17$ & $53-40 \mathrm{~min}$ \\
8 & $17: 21$ & $40-32 \mathrm{~min}$ \\
9 & $21: 26$ & $32-27 \mathrm{~min}$ \\
10 & $26: 31$ & $27-21 \mathrm{~min}$ \\
\hline
\end{tabular}

Spectral power from $24 \mathrm{~h}$ of CSF samples with 10 min intersample interval, $n=13$. Decline in 5-HIAA spectral power density reflects decreased variance after treatment, and increased HVA and ratio spectral power density (HVA: 5 HIAA) reflect increased variance with treatment.

Distribution of PSD coefficients across 10 bandwidths used for spectrogram statistical analysis (see Figure 2). Note that coefficient density was preserved across bandwidths by disregarding the last two coefficients (32 and 33), effectively shortening the period range by only $0.5 \mathrm{~min} / \mathrm{cycle}$, ending instead at $20.5 \mathrm{~min} /$ cycle.

robust change was seen across all discernable subject subsets (gender, age, severity, treatment response, and antidepressant treatment group) in this set of nongeriatric adult depressed patients, and was observed in a continuous cluster of significant points through an uninterrupted block of time.

\section{Medication Treatment}

Overall, antidepressant treatments yielded a mood response in $62 \%$ of the subjects (sertraline $6 / 9$ treated, bupropion $2 / 4$ treated), who also met criteria for remission. Severity at baseline may have partly predicted remission but was not statistically significant (responders' baseline HRSD $x=18.3 \pm 4.6$; nonresponders' baseline HRSD $x=20.3 \pm 3.1)$. No statistically significant relationship between mood response and change in spectral power was detected in this small group.

Both drug groups, both response groups, and both gender groups displayed similarly enhanced variance of the HVA : 5-HIAA ratio in the ca. $84 \mathrm{~min}(1.2-1.7 \mathrm{~h})$ frequency bandwidth. The data set is clearly underpowered to detect any subtle difference in these groupings, and no clear trends were found. Some subset analyses are given in Supplementary material available online, demonstrating the acceptability of merging treatment and response groups for analysis.

\section{Metabolite Level Means, Correlations, and Variances}

Means of each sample series were compared ( $n=13$ pairs). CSF 5-HIAA levels dropped by $29 \%$ with treatment, from $119.7 \pm 28.6 \mathrm{nM} / 1$ pretreatment to $85.1 \pm 27.7 \mathrm{nM} / 1$ posttreatment (means of 1872 samples; 24-h collections, 144 samples from 13 patients; as paired $t$-tests $n=13$, $t(12)=3.32, p=0.006$ ). For comparison purposes, healthy control CSF 5-HIAA levels collected in previous studies under similar conditions were higher than the depressed baseline and much higher than the post-treatment measures ( $136 \%$ of depressed values, $163.0 \pm 50.2 \mathrm{nM} / \mathrm{l}, n=12 \times 144$ samples).

Mean HVA levels in the depressed sample were virtually unchanged from pretreatment $(280.1 \pm 100.4)$ to posttreatment $(283.1 \pm 78.5)$. Mean ratios of HVA:5-HIAA changed proportionately with the change in the denominator values, from $2.34 \pm 0.55$ pretreatment to $3.46 \pm 0.99$ after treatment (paired $t(12)=-4.23, p=0.001$ ). The measurements appear to be valid, as they agreed in direction and magnitude with changes in mean metabolite levels from single-tap, larger population studies of antidepressant treatment effects.

Correlations between 5-HIAA and HVA post-treatment $(r=0.39)$ were weaker than pretreatment $(r=0.70)$, suggesting a possible increase in independence of the two neurotransmitter systems with treatment (Pearson-Filon $z$ score (Raghunathan et al, 1996), $\mathrm{ZPF}=1.56, p=0.06$ ). Given this putative increase in independence between these systems with treatment, the time series data were examined for changes in variance and sources of the variance. Statistical variance (averaging individual variance across subjects) of 5-HIAA, a measure replicated by the AUC of the PSDs (Table 1), decreased by $44 \%$ overall with treatment, while data from the same assays showed increased variances of HVA and of the HVA:5-HIAA ratio by 81 and 243\%, respectively. Variance of simultaneously measured CSF tryptophan (TRP, the precursor of serotonin) was unchanged, providing a negative control (Table 1) and

\section{Smoothed Ratio PSDs Change with Treatment}

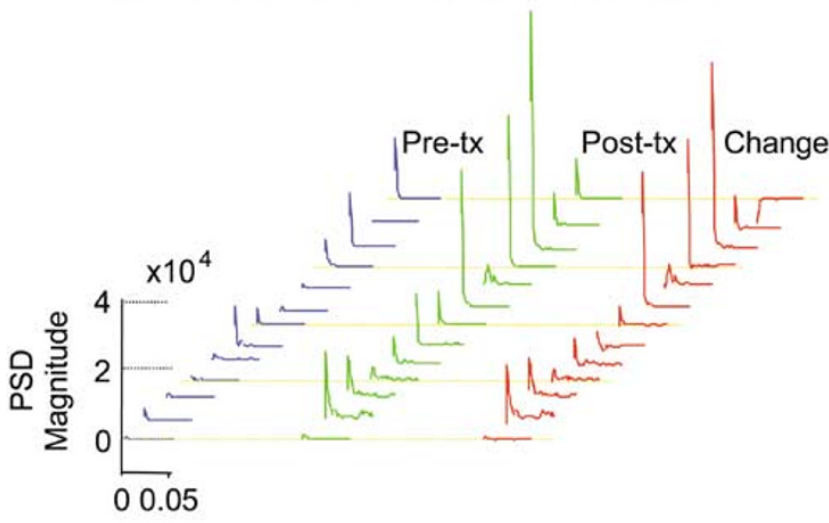

Frequency (cycles per minute)

Figure I 24-h PSD of HVA:5-HIAA ratio. Change with treatment in PSDs of the CSF HVA: 5-HIAA ratio from 24-h serial data (sampled every $10 \mathrm{~min}$ ), showing marked within-subject changes for 13 depressed patients, corresponding to the means summarized in Table 2. The horizontal axis (log (frequency); cycles/min), runs from one cycle per $24 \mathrm{~h}$ (near the origin) to one cycle per $20 \mathrm{~min}$ ( $=0.05$ cycles per $\mathrm{min}$ ). The halfway point is at about $100 \mathrm{~min} / \mathrm{cycle}$. Vertical axis reflects PSD power with local smoothing for clarity. The three series of individual plots for each patient are pretreatment, post-treatment, and their difference (post- minus pre-), respectively, from left to right, with each data from patient aligned in sequence. Power is consistently elevated at low frequencies, shown near the origin of each PSD, with lower power at high frequencies $(20 \mathrm{~min}$ periods) at the far right for each curve. The PSD is the square of Fourier transform coefficients. Identical axis scaling was used for every plot. 
supporting an assertion that the sample collection method and HPLC methods were not likely sources of artefact that could explain the variance changes in other measures.

\section{Fourier Analysis and Spectrograms}

Spectral analysis (Table 2) of the full-length series of 5HIAA levels following treatment showed significantly reduced power in low frequencies (long periods, $3.75 \mathrm{~h} /$ cycle) and midrange frequencies (medium periods, $1.75 \mathrm{~h} /$ cycle) compared with pretreatment values. In contrast, the HVA signal (still full length) showed increased power predominantly at the lowest detectable frequency $(24 \mathrm{~h} /$ cycle). Spectral power of the HVA:5-HIAA ratio (Figure 1) increased in both the lowest $(24 \mathrm{~h} /$ cycle $)$ and high $(0.75 \mathrm{~h} /$ cycle, or $45 \mathrm{~min} /$ cycle) frequencies (again, calculated over the entire sample).

Spectrograms, serially examining $8.7 \mathrm{~h}$ data windows through the day (and zero-padded to give a first (nonDC) coefficient at $10.7 \mathrm{~h}$ periods) were divided into 10 frequency bandwidths to cluster nearby frequencies in a single analysis (Table 3, Figure 2). The ratio data showed changes in only the fifth bandwidth (1.2-1.7 h/cycle, centered at $84 \mathrm{~min} /$ cycle) after correction for multiple comparisons. Treatment was associated with significantly increased spectral power of the HVA:5-HIAA ratio in the evening hours, with peak power at 2100 hours for this bandwidth (17 adjacent windows totaling $2.8 \mathrm{~h}$, significant $t$-values from 3.54 to 4.00 ; effect size, $d \geqslant 2.04 ; p<0.05$ by MPT, Figure 2c). The centers of this block of windows spanned the evening from 1910 to 2150 hours with window

a

$t$ values for spectral change with treatment: CSF 5-HIAA

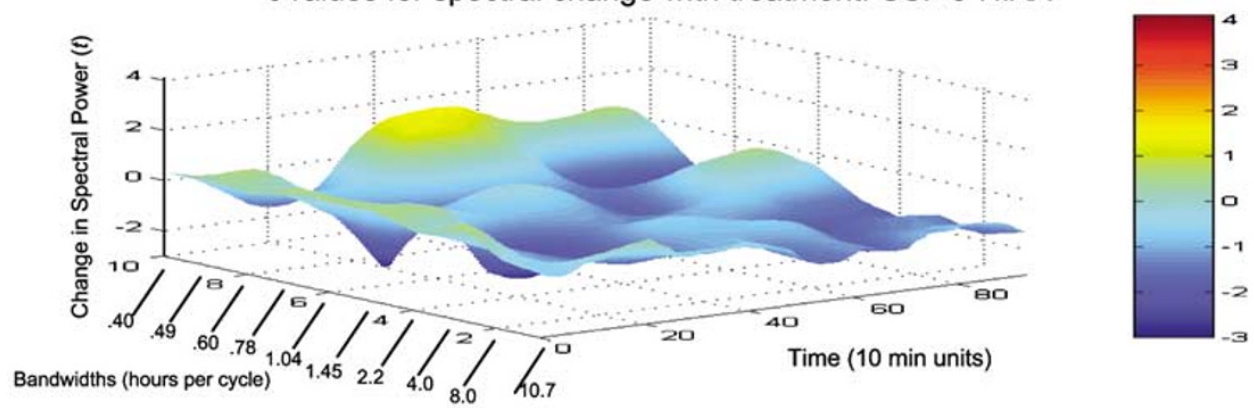

b

$\boldsymbol{t}$ values for spectral change with treatment: CSF HVA

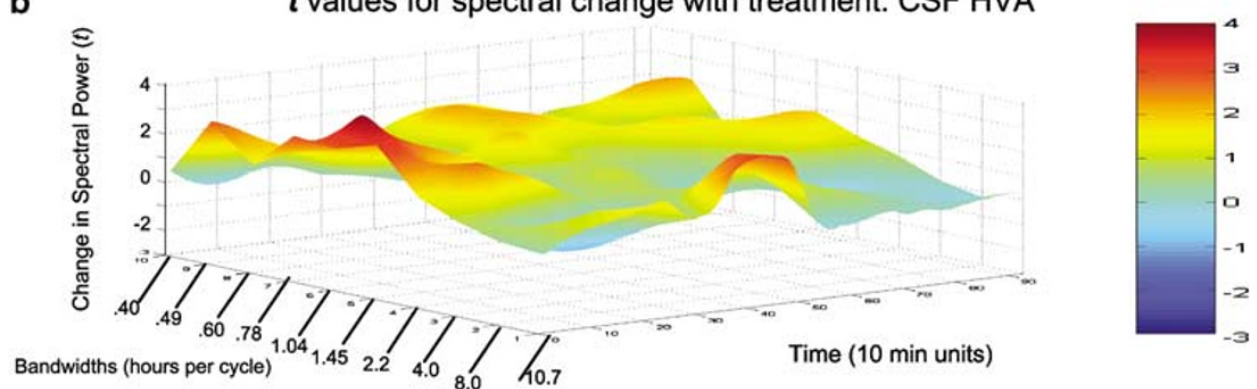

C

$\boldsymbol{t}$ values for spectral change with treatment: CSF HVA:5-HIAA Ratio

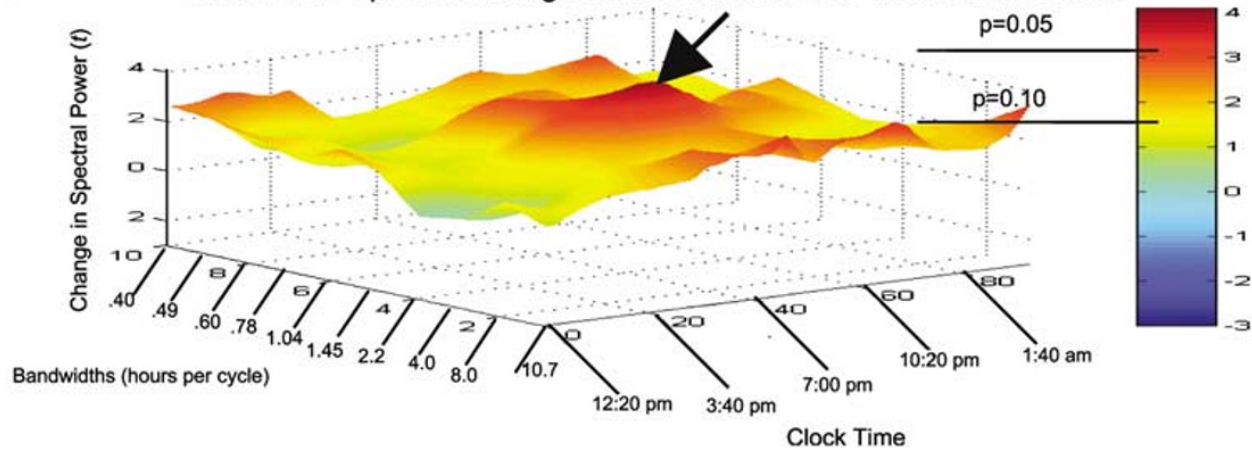

Figure 2 Spectrograms of change in CSF monoamine metabolite oscillations. Treatment-associated $t$-values for changes in spectral density (PSD) using 520-min windows through the 24-h period after 5 weeks treatment in 13 patients to form spectrograms. The spectrogram displays PSDs from overlapping windows along the time axis. Time axis labels are window centers, with clock times for all plots as marked on the bottom plot. Frequency axis bandwidths run from $10.7 \mathrm{~h}$ per cycle (band I) to $20 \mathrm{~min}$ per cycle (band I0), see Table 3. (a) 5-HIAA: Strongest change was a decline in PSD power at midrange frequencies in the early evening. (NS). (b) HVA: Strongest change was a rise in PSD power at midrange frequencies (bandwidth 6) between noon and I 300 hours (trend significance for 3 points, $d f=12$, with $t$-values 3.96-4.07; effect size, $d \geqslant 2.29 ; p<0.10$ ). (c) Significantly increased HVA: 5-HIAA ratio oscillatory power associated with antidepressant treatment. Arrow marks a peak at 84-min periods at 2100 hours, significant elevation across 17 adjacent windows, lasting $2.8 \mathrm{~h}$ (significant $t$-values from 3.54 to 4.00 ; effect size $d \geqslant 2.04$; $p<0.05$ by MPT). 
margins extending from 1450 to 0210 hours. This change in the ratio spectrum was not simply due to changes in either of the 5-HIAA or HVA mean spectra since neither showed significant changes in the spectrogram function with treatment (Figures 2a and b). The MPT method corrects for the number of comparisons conducted in the single test.

\section{DISCUSSION}

To our knowledge, this is the first report to show ultradian rhythms in the ratio of HVA:5-HIAA or changes in those rhythms with treatment for depression. Antidepressant treatment was associated with increased spectral power of the HVA:5-HIAA ratio for frequencies in a bandwidth around $84 \mathrm{~min}$, and lasting $2.8 \mathrm{~h}$ in the evening. Oscillatory changes were less consistent in the individual monoamine measures (HVA or 5-HIAA) than in their ratio, possibly because the ratio analysis detected true changes in interactions between the systems.

Enhanced variability in the HVA:5-HIAA ratio after treatment suggests an increase in the functional independence of these aminergic systems whether by increased negative interactions, decreased positive interaction, or diminished external synchronization. This raises questions regarding mood regulation and etiology of depression. In the current literature, no single transmitter or transmitterrelated mechanism has been conclusively linked to depression, but greater antidepressant efficacy has been observed with agents that affect multiple neurotransmitter mechanisms (for a review, see Tran et al, 2003), or in strategies that employ more frequent medication changes (Trivedi et al, 2004). Two different antidepressant classes were equally potent for memory and affective perceptions in healthy volunteers (Harmer et al, 2004). While seemingly counterintuitive, ceasing treatments that have lost efficacy often results in temporary mood improvement. Taken together, these observations suggest that selectively altering the function of one component of an interactive network of transmitter systems may serve to alleviate maladaptively 'locked' patterns that interfere with healthy function.

Locked patterns are also observed behaviorally throughout the depression diagnostic subtype spectrum. Depression subtypes show excessively fixed symptoms at extremes of function (for example, overactive or inhibited appetite). Melancholic severity and elevated suicidality (Grunebaum et al, 2004) have been related to defective second messenger systems coupled to the-serotonin-2a receptor (Akin et al, 2004), a mechanism that profoundly alters physiologic variability. Melancholia disrupts anterior cingulate function (Rogers et al, 2004), a correlate of mental slowness and cognitive deficits that also represent a lack of functional variability. Patients with atypical depression endure fixed anger and extreme sadness, while those with seasonal affective disorder who fixed low mood and poor energy. It may be that invariant interactions between transmitter systems fix pathogenic network behaviors, in part by constraining stress responses. Regulators that control variability of system interactions, such as clock genes or other metabolic switching mechanisms, may present a future target for depression treatments.
Previous studies examining similar CSF time series data did not report changes in oscillatory function, most likely because of shorter and less frequent sampling. Healthy subjects, sampled every $30 \mathrm{~min}$ for $6 \mathrm{~h}$, did not show oscillations in the HVA:5-HIAA ratio (Geracioti et al, 1998). In depression, an uncoupling of 5-HT and norepinephrine was suggested based on the absence of a negative linear relationship that was present in healthy subjects after $6 \mathrm{~h}$ sampling (Geracioti et al, 1997). More intensive and longer collections would be helpful.

The literature provides no similar comparison data sets, wherein one could assess subgroups (gender, drug mechanism, or treatment response) in patients undergoing CSF measurements with a prolonged time series. However, in one study, variance in CSF metabolites was affected by the presence or absence of premenstrual symptoms. Single CSF samples taken during luteal and follicular phases were compared in 'PMS' and control patients, and showed less intrasubject variability in CSF levels of HVA and 5-HIAA in the 'PMS' group (Eriksson et al, 1994). Accurate last menstrual period data and assessments of late luteal symptom changes are not available for the current population sample, and women who were postmenopausal or posthysterectomy were included, thus limiting the number available for direct comparison to the Eriksson study. Studies currently under way are limited to subjects under age 50 years, in part in order to address this issue.

The findings reported here are not meant to suggest a singular, exclusive effect of antidepressants on any single transmitter system or dynamical measure. Collection and analysis conditions were poised to detect rhythmic periods between $20 \mathrm{~min}$ and $24 \mathrm{~h}$, with the longest period dependent on window length in the spectrogram (about $10 \mathrm{~h}$ in our analysis). A limited number of substances have been assayed. Moreover, it is likely that there are regionally specific rhythms that are obscured or weakened by the global nature of lumbar sampling. Caveats remain that the present ratio analysis cannot clearly distinguish between, for example, enhanced variability in HVA and dampening of variability in 5-HIAA, and that the changes observed with antidepressant treatment might be due to artefacts of medication that are unrelated to mood pathophysiology.

Finding periodicity changes in the HVA:5-HIAA ratio but not in the single metabolite levels could be related to factors other than true system interactions that are discussed above. Combining data from two antidepressant treatments (sertraline and bupropion) with different treatment mechanisms, or a masking of signal noise in the HVA : 5-HIAA ratio that obscures a signal in the individual metabolite series might also explain the isolation of the finding in the ratio data. The absence of enhancement later in the evening and through the night might have been influenced by less than ideal sleep conditions, given the frequent room entries for sample retrieval and vital sign monitoring. The consistent absence of the 90-min oscillations in the evening during the pretreatment collection may herald an abnormal loss of variability in depression. It should be noted that the use of clustered frequency coefficients in the analysis can be justified since strict rhythms of nocturnal REM cycles are not expected; in EEG data, $95 \%$ of the distribution would require REM onset periodicity to range from 50 to $130 \mathrm{~min}$ (Bennington, 1999). 
Heuristically, a rhythm-driven conceptualization of mood dysfunction is evident in clinical practice, where depressed patients frequently complain of their 'stuckness' - a symptom that is not part of our routine diagnostic criteria. Physiologically, blunted neuroendocrine responses may be a reflection of 'stuckness', not just of the endocrine output but also of the monoaminergic inputs. In this view, neural network rhythms would need to be healthy in order to maintain healthy network functional output. Fluctuations in transmitter activity may confer health and flexibility in neural network function, and this may be a critical aspect of neurochemical dysfunction in depression. To find genes that control ultradian rhythms, strengths of these signals will need to be measured with time series analysis in the frequency domain. Neurochemical systems are unlikely to project perfect circles onto maps of their orbits. Complex attractors represent imperfect oscillators within bounded orbits, modeling periodic functions common in nature. Oscillatory cycling (Hobson et al, 1975) and chaotic attractors (McCarley and Massaquoi, 1986) modeled REM on the basis of REM-on and REM-off neuronal activities. The argument regarding the existence of ultradian oscillators is far from closed, as it is becoming increasingly difficult to refute the likelihood of a genetic regulation of sleep physiology and its ultradian markers. The recognition of regulators of neurochemical ultradian mood behavior may not be far behind.

In conclusion, these data present evidence from a small number of patients of an antidepressant effect on ultradian rhythms in central neurochemical time series measures in human depression. Enhancements of transmitter system independence in near REM-cycle frequencies may be a hallmark of treatment effects. Further study may uncover genetic timers that control ultradian regulatory variability for mood circuitry, even if strictly precise rhythms are not evident. The relationship between depression and a periodic variability in transmitter system independence merits further study.

\section{ACKNOWLEDGEMENTS}

We are particularly grateful to all of the subjects and GCRC staff, and for helpful discussions and support from Richard Shelton, MD, and Ariel Deutch, PhD among many others. This work was funded by grant support to RMS (K23 MH01828 from NIMH, the Stanley Foundation, NARSAD, and an investigator-initiated grant from Pfizer, Inc.), to the Vanderbilt General Clinical Research Center (M01 RR00095 from NICCR), and to the Vanderbilt Kennedy Center for Research on Human Development (supporting JUB, grant P30 HD15052 from NICHD). Presented, in part, in meeting abstracts: the American Psychiatric Association, Toronto May 1998, and the American College of Neuropsychopharmacology, Puerto Rico December 1998 and December 2003.

\section{REFERENCES}

Agren H, Koulu M, Saavedra JM, Potter WZ, Linnoila M (1986). Circadian covariation of norepinephrine and serotonin in the locus coeruleus and dorsal raphe nucleus in the rat. Brain Res 397: 353-358.
Akin D, Manier DH, Sanders-Bush E, Shelton RC (2004). Decreased serotonin 5-HT2A receptor-stimulated phosphoinositide signaling in fibroblasts from melancholic depressed patients. Neuropsychopharmacology 29: 2081-2087.

American Psychiatric Association Diagnostic and Statistical Manual of Mental Disorders, 4th edn. APA: Washington, DC; 1994.

Armitage R, Emslie GJ, Hoffmann RF, Weinberg WA, Kowatch RA, Rintelmann J et al (2000). Ultradian rhythms and temporal coherence in sleep EEG in depressed children and adolescents. Biol Psychiatry 47: 338-350.

Armitage R, Hoffman RF, Rush AJ (1999). Biological rhythm disturbance in depression: temporal coherence of ultradian sleep EEG rhythms. Psychol Med 29: 1435-1448.

Bennington JH (1999). Why we believe what we believe about REM-sleep regulation. In: Mallick BN, Inoue S (eds). Rapid Eye Movement Sleep. Narosa Publishing House: New Delhi, India. pp 393-401 quoting Lewis S (1974): The paradoxical sleep cycle revisited. In: Chronobiology Igaku Shoin: Tokyo. pp 487-490.

Birkmayer W, Linauer W (1970). Störung des Tyrosin- und Tryptophan- metabolismus bei Depression. Arch Psychiat Nervenkr 213: 377-387.

Blackford J (2002). Multivariate Permutation Testing for Paired $t$-Tests (computer program), Version 1.2. Vanderbilt Kennedy Center for Research in Human Development: Nashville, TN. (Requires R Project statistical software.).

Blair RC, Karniski W (1994). Distribution-free statistical analyses of surface and volumetric maps. In: Thatcher RW, Hallet M, Zeffiro T, John ER, Huerta M (eds). Functional Neuroimaging: Technical Foundations. Academic Press: San Diego.

Blier P, Galzin AM, Langer SZ (1989). Diurnal variation in the function of serotonin terminals in the rat hypothalamus. J Neurochem 52: 453-459.

Bunney WE, Bunney BG (2000). Molecular clock genes in man and lower animals: possible implications for circadian abnormalities in depression. Neuropsychopharmacology 22: 335-345.

Carpenter LL, Kupfer DJ, Frank E (1986). Is diurnal variation a meaningful symptom in unipolar depression? J Affect Disord 11: 255-264

Carpenter LL, Moreno FA, Kling MA, Anderson GM, Regenold WT, Labiner DM et al (2004). Effect of vagus nerve stimulation on cerebrospinal fluid monoamine metabolites, norepinephrine, and gamma-aminobutyric acid concentrations in depressed patients. Biol Psychiatry 56: 418-426.

Cassano P, Fava M (2004). Tolerability issues during long-term treatment with antidepressants. Ann Clin Psychiatry 16: 15-25.

Cazard P, Ricard F, Facchetti L (1992). Depression and functional EEG asymmetry. Ann Med Psychol (Paris) 150: 230-239.

Chergui K, Svenningsson P, Greengard P (2004). Cyclin-dependent kinase 5 regulates dopaminergic and glutamatergic transmission in the striatum. Proc Natl Acad Sci USA 101: 2191-2196 Epub 2004 Feb 09.

Cohen J (1992). A power primer. Psychol Bull 112: 155-159.

DeBellis MD, Geracioti Jr TD, Altemus M, Kling MA (1993). Cerebrospinal fluid monoamine metabolites in fluoxetinetreated patients with major depression and in healthy volunteers. Biol Psychiatry 33: 636-641.

Duncan Jr WC (1986). Circadian rhythms and the pharmacology of affective illness. Pharmacol Ther 71: 243-312.

Duncan Jr WC, Johnson KA, Sutin E, Wehr TA (1998). Disruption of the activity-rest cycle by MAOI treatment: dependence on light and a secondary visual pathway to the circadian pacemaker. Brain Res Bull 45: 457-465.

Ehlers CL, Frank E, Kupfer DJ (1988). Social zeitgebers and biological rhythms. A unified approach to understanding the etiology of depression. Arch Gen Psychiatry 45: 948-952.

Ehlers CL, Kupfer DJ (1987). Hypothalamic peptide modulation of EEG sleep in depression: a further application of the S-process hypothesis. Biol Psychiatry 22: 513-517. 
Engstrom G, Alling C, Blennow K, Regnell G, Traskman-Bendz L (1999). Reduced cerebrospinal HVA concentrations and HVA/5HIAA ratios in suicide attempters. Monoamine metabolites in 120 suicide attempters and 47 controls. Eur Neuropsychopharmacol 5: 399-405.

Eriksson E, Alling C, Andersch B, Andersson K, Berggren U (1994). Cerebrospinal fluid levels of monoamine metabolites. A preliminary study of their relation to menstrual cycle phase, sex steroids, and pituitary hormones in healthy women and in women with premenstrual syndrome. Neuropsychopharmacology 11: 201-213.

Fabre V, Boutrel B, Hanoun N, Lanfumey L, Fattaccini CM, Demeneix B et al (2000). Homeostatic regulation of serotonergic function by the serotonin transporter as revealed by nonviral gene transfer. J Neurosci 20: 5065-5075.

First MB, Spitzer RL, Gibbon M, Williams JBW (1996a). Structured Clinical Interview for DSM-IV Axis I Disorders (SCID-I/P), (Version 20). Biometrics Research Department, New York State Psychiatric Institute, 722 West 168th Street: NY 10032, USA.

First MB, Spitzer RL, Gibbon M, Williams JBW, Benjamin L (1996b). Structured Clinical Interview for DSM-IV Axis II Personality Disorders (SCID-II), (Version 20) Biometrics Research Department, New York State Psychiatric Institute, 722 West 168th Street: NY 10032, USA.

Geracioti Jr TD, Keck Jr PE, Ekhator NN, West SA, Baker DG, Hill KK et al (1998). Continuous covariability of dopamine and serotonin metabolites in human cerebrospinal fluid. Biol Psychiatry 44: 228-233.

Geracioti Jr TD, Loosen PT, Ekhator NN, Schmidt D, Chambliss B, Baker DG et al (1997). Uncoupling of serotonergic and noradrenergic systems in depression: preliminary evidence from continuous cerebrospinal fluid sampling. Depress Anxiety 6: 8994.

Geracioti Jr TD, Orth DN, Ekhator NN, Blumenkopf B, Loosen PT (1992). Serial cerebrospinal fluid corticotropin-releasing hormone concentrations in healthy and depressed humans. J Clin Endocrinol Metab 74: 1325-1330.

Grunebaum MF, Galfalvy HC, Oquendo MA, Burke AK, Mann JJ (2004). Melancholia and the probability and lethality of suicide attempts. Br J Psychiatry 184: 534-535.

Harmer CJ, Shelley NC, Cowen PJ, Goodwin GM (2004). Increased positive versus negative affective perception and memory in healthy volunteers following selective serotonin and norepinephrine reuptake inhibition. Am J Psychiatry 161: 1256-1263.

Haug H-J, Wirz-Justice A (1993). Diurnal variation of mood in depression: important or irrelevant? Biol Psychiatry 34: 201-203.

Hayashi M, Sato K, Hori T (1994). Ultradian rhythms in task performance, self-evaluation, and EEG activity. Percept Mot Skills 79: 791-800.

Healy D (1987). Rhythm and blues. Neurochemical, neuropharmacological and neuropsychological implications of a hypothesis of circadian rhythm dysfunction in the affective disorders. Psychopharmacology (Berl) 93: 271-285.

Hill KK, West SA, Ekhator NN, Bruce AB, Wortman MD, Baker DG et al (1999). The effect of lumbar puncture stress on dopamine and serotonin metabolites in human cerebrospinal fluid. Neurosci Lett 276: 25-28.

Hobson JA, McCarley RW, Wyzinski PW (1975). Sleep cycle oscillation: reciprocal discharge by two brainstem neuronal groups. Science 189: 55-58.

Holmes MC, French KL, Seckl JR (1997). Dysregulation of diurnal rhythms of serotonin 5-HT2C and corticosteroid receptor gene expression in the hippocampus with food restriction and glucocorticoids. J Neurosci 17: 4056-4065.

Jouvent R, Le Houezec J, Payan C, Mikkelsen H, Fermanian J, Millet V et al (1998). Dimensional assessment of onset of action of antidepressants: a comparative study of moclobemide vs clomipramine in depressed patients with blunted affect and psychomotor retardation. Psychiatry Res 79: 267-275.

Kennedy JS, Gwirtsman HE, Schmidt DE, Johnson BW, Fielstein E, Salomon RM et al (2002). Serial cerebrospinal fluid tryptophan and 5-hydroxy indoleacetic acid concentrations in healthy human subjects. Life Sci 71: 1703-1715.

Klein R, Armitage R (1979). Rhythms in human performance: 1 1/ 2-hour oscillations in cognitive style. Science 204: 1326-1328.

Kleitman N (1982). Basic rest-activity cycle -22 years later. Sleep 5: $311-317$.

Knapp S, Mandell AJ (1983). Lithium and chlorimipramine differentially alter the stability properties of tryptophan hydroxylase as seen in allosteric and scattering kinetics. Psychiatry Res 8: $311-323$.

Knott VJ, Telner JI, Lapierre YD, Browne M, Horn ER (1996). Quantitative EEG in the prediction of antidepressant response to imipramine. J Affect Disord 39: 175-184.

Kraepelin E (1913).. Klinische Psychiatrie. Ein Lehrbuch für Studierende und Ärzte. III. Be., II. Teil. Verlag J.A. Barht, Leipzig.

Kulikov A, Mormede P, Chaouloff F (1997). Effects of adrenalectomy and corticosterone replacement on diurnal [3H]-citalopram binding in the rat midbrain. Neurosci Let 222: 127-131.

Lovenberg TW, Baron BM, de Lecea L, Miller JD, Prosser RA, Rea MA et al (1993). A novel adenylyl cyclase-activating serotonin receptor (5-HT7) implicated in the regulation of mammalian circadian rhythms. Neuron 11: 449-458.

Mazure C, Nelson JC, Price LH (1986). Reliability and validity of the symptoms of major depressive illness. Arch Gen Psychiatry 43: 451-456.

McCarley RW, Massaquoi SG (1986). A limit cycle mathematical model of the REM sleep oscillator system. Am J Physiol Regul Integr Comp Physiol 251: R1011-R1029.

Mullins UL, Gianutsos G, Eison AS (1999). Effects of antidepressants on 5-HT7 receptor regulation in the rat hypothalamus. Neuropsychopharmacology 21: 352-367.

Nakayama K (2002). Diurnal rhythm in extracellular levels of 5hydroxyindoleacetic acid in the medial prefrontal cortex of freely moving rats: an in vivo microdialysis study. Prog Neuropsyhopharmacol Biol Psychiatry 26: 1383-1388.

Nishi M, Kawata M, Azmitia EC (2000). Trophic interactions between brain-derived neurotrophic factor and s100beta on cultured serotonergic neurons. Brain Res 868: 113-118.

Oxenkrug GF (1979). The content and uptake of 5-HT by blood platelets in depressive patients. J Neural Transm 45: 285-289.

Pezard L, Nandrino JL, Renault B, el Massioui F, Allilaire JF, Muller J et al (1996). Depression as a dynamical disease. Biol Psychiatry 39: 991-999.

Polich J (1997). On the relationship between EEG and P300: individual differences, aging, and ultradian rhythms. Int $J$ Psychophysiol 26: 299-317.

Prospero-Garcia O, Navarro L, Murillo-Rodriguez E, SanchezAlvarez M, Guzman-Marin R, Mendez-Diaz M et al (1999). Cellular and molecular changes occurring during REM sleep. In: Mallick BN, Inoue S (eds). Rapid Eye Movement Sleep. Narosa Publishing House: New Delhi, India. pp 367-381.

R: Copyright 2003. The R Foundation for Statistical Computing. Version 1.8.1 (2003-11-21), ISBN 3-900051-00-3.

Raghunathan TE, Rosenthal R, Rubin DB (1996). Comparing correlated but nonoverlapping correlations. Psychol Methods 1: 178-183.

Rao ML, Groww G, Strebel B, Halaris A, Huber G, Bräunig P et al (1994). Circadian rhythm of tryptophan, serotonin, melatonin, and pituitary hormones in schizophrenia. Biol Psychiatry 35: 151-163.

Raoux N, Benoit O, Dantchev N, Denise P, Franc B, Allilaire JF et al (1994). Circadian pattern of motor activity in major depressed patients undergoing antidepressant therapy: relationship bet- 
ween actigraphic measures and clinical course. Psychiatry Res 52: $85-98$.

Riederer P, Birkmayer W, Neumayer E, Ambrozi L, Linauer W (1974). The daily rhythm of HVA, VMA, (VA) and 5-HIAA in depression-syndrome. J Neural Transm 35: 23-45.

Risby ED, Hsiao JK, Sunderland T, Agren H, Rudorfer MV, Potter WZ (1987). The effects of antidepressants on the cerebrospinal fluid homovanillic acid/5-hydroxyindoleacetic acid ratio. Clin Pharmacol Ther 42: 547-554.

Rogers MA, Bellgrove MA, Chiu E, Mileshkin C, Bradshaw JL (2004). Response selection deficits in melancholic but not nonmelancholic unipolar major depression. J Clin Exp Neuropsychol 26: 169-179.

Salomon RM, Ripley B, Kennedy JS, Johnson B, Schmidt D, Zeitzer JM et al (2003). Diurnal variation of cerebrospinal fluid hypocretin-1 (Orexin-A) levels in control and depressed subjects. Biol Psychiatry 54: 96-104.

Schildkraut JJ (1965). The catecholamine hypothesis of affective disorders: a review of supporting evidence. Am J Psychiatry 122: 509-522.

Schmidt DE, Roznoski ML, Ebert MH (1990). Qualitative and quantitative HPLC analysis of monoamine neurotransmitters and metabolites in CSF and brain tissue using reductive electrochemical detection. Biomed Chromotogr 4: 215.

Shannahoff-Khalsa D (1993). The ultradian rhythm of alternating cerebral hemispheric activity. Int J Neurosci 70: 285-298.

Thase ME, Fasiczka AL, Berman SR, Simons AD, Reynolds III CF (1998). Electroencephalographic sleep profiles before and after cognitive behavior therapy of depression. Arch Gen Psychiatry 55: $138-144$.

Tran PV, Bymaster FP, McNamara RK, Potter WZ (2003). Dual monoamine modulation for improved treatment of major depressive disorder. J Clin Psychopharmacol 23: $78-86$.

Trivedi MH, Rush AJ, Crismon ML, Kashner TM, Toprac MG, Carmody TJ et al (2004). Clinical results for patients with major depressive disorder in the Texas Medication Algorithm Project. Arch Gen Psychiatry 61: 669-680.

Van Reeth O, Olivares E, Zhang Y, Tripathi B, Turek FW (1999). Chronobiotic effects of gepirone, a potential antidepressant with 5HT1A receptor partial agonist properties. Behav Pharmacol 10: 119-130.

Wefelmeyer T, Kuhs H (1996). Diurnal mood variation in melancholic patients and healthy controls. Psychopathology 29: 184-192.

Wehr TA, Muscettola G, Goodwin FK (1980). Urinary 3-methoxy4-hydroxyphenylglycol circadian rhythm. Early timing (phaseadvance) in manic-depressives compared with normal subjects. Arch Gen Psychiatry 37: 257-263.

Wehr TA, Wirz-Justice A, Goodwin FK, Duncan W, Gillin JC (1979). Phase advance of the circadian sleep-wake cycle as an antidepressant. Science 206: 710-713.

Wirz-Justice A (2000). Biological rhythms in mood disorders. In: FE Bloom and DE Kupfer (eds). Neuropsychopharmacology: the Fourth Generation of Progress http://www.acnp.org/g4/ GN401000099/Default.htm accessed 1/9/05.

Supplementary Information accompanies the paper on Neuropsychopharmacology website (http://www.nature.com/npp) 